\title{
Molecular Phylogeny of the Genus Frankia and Related Genera and Emendation of the Family Frankiaceae
}

\author{
PHILIPPE NORMAND, ${ }^{1 *}$ STÉPHANIE ORSO, ${ }^{1}$ BENOIT COURNOYER, ${ }^{1}$ PASCALE JEANNIN, ${ }^{2}$ \\ CHRYSTELLE CHAPELON, ${ }^{1}$ JEFFREY DAWSON,${ }^{3}$ LYSE EVTUSHENKO, ${ }^{4}$ \\ AND ARVIND K. MISRA \\ Ecologie Microbienne du Sol, URA CNRS 1977, Université Claude-Bernard, Lyon 1, 69622 Villeurbanne Cedex, ${ }^{1}$ \\ and Rhône-Poulenc Rorer, Vitry-sur-Seine, ${ }^{2}$ France; Department of Forestry, University of Illinois, \\ Urbana, Illinois 61801; ${ }^{3}$ Pushchino Institute, Moscow, Russia ${ }^{4}$; and Department of \\ Botany, North-Eastern Hill University, Shillong, India ${ }^{5}$
}

\begin{abstract}
The members of the actinomycete genus Frankia are nitrogen-fixing symbionts of many species of woody dicotyledonous plants belonging to eight families. Several strains isolated from diverse actinorhizal plants growing in different geographical areas were used in this study. The phylogenetic relationships of these organisms and uncharacterized microsymbionts that are recalcitrant to isolation in pure culture were determined by comparing complete $16 \mathrm{~S}$ ribosomal DNA sequences. The resulting phylogenetic tree revealed that there was greater diversity among the Alnus-infective strains than among the strains that infect other host plants. The four main subdivisions of the genus Frankia revealed by this phylogenetic analysis are (i) a very large group comprising Frankia alni and related organisms (including Alnus rugosa $\mathrm{Sp}+$ microsymbionts that are seldom isolated in pure culture), to which Casuarina-infective strains, a Myrica nagi microsymbiont, and other effective Alnus-infective strains are related; (ii) unisolated microsymbionts of Dryas, Coriaria, and Datisca species; (iii) Elaeagnus-infective strains; and (iv) "atypical" strains (a group which includes an Alnus-infective, non-nitrogen-fixing strain). Taxa that are related to this well-defined, coherent Frankia cluster are the genera Geodermatophilus, "Blastococcus," Sporichthya, Acidothermus, and Actinoplanes. However, the two genera whose members have multilocular sporangia (the genera Frankia and Geodermatophilus) did not form a coherent group. For this reason, we propose that the family Frankiaceae should be emended so that the genera Geodermatophilus and "Blastococcus" are excluded and only the genus Frankia is retained.
\end{abstract}

The slowly growing members of the actinomycete genus Frankia are root symbionts that nodulate a wide range of perennial woody dicotyledonous plants. This nitrogen-fixing symbiosis is known to occur in more than 200 species of plants belonging to 24 genera and eight families that are called actinorhizal (6). The first Frankia strain was isolated in 1956 by Pommer (43), but this strain was subsequently lost. In 1978, Callaham et al. (8) isolated an infective Frankia strain from Comptonia peregrina, and since then hundreds of isolates have been obtained from a number of plant species growing in many geographical areas.

Becking (5) was unsuccessful in isolating the causative agent of actinorhizal nodules despite numerous attempts. He suggested that this organism was an "obligate symbiont" and devised a classification scheme based on cross-inoculation groups and on the morphology of the endosymbiont. This scheme was subsequently found to be erroneous when pure cultures became available (8). Nonetheless, Becking (5) correctly perceived the bacterial nature of the microsymbiont, named it Frankia sp., and classified it as the only member of the family Frankiaceae in the order Actinomycetales.

The members of the genus Frankia can now be clearly distinguished from other bacterial genera on the basis of their host specificity, their morphology (hyphae, a specialized thickwalled organelle called a diazovesicle, and multilocular sporangia containing nonmotile spores), their biochemistry (a type

\footnotetext{
${ }^{*}$ Corresponding author. Mailing address: Ecologie Microbienne du Sol, URA CNRS 1977, 43 bd du 11 novembre 1918, Université ClaudeBernard, Lyon 1, 69622 Villeurbanne Cedex, France. Phone: 724313 77. Fax: 724312 23. Electronic mail address: normand@ecosol.univlyon1.fr.
}

III cell wall that contains meso-diaminopimelic acid, glutamic acid, alanine, glucosamine, and muramic acid and type PI phospholipids that include phosphatidylinositol, phosphatidylinositol mannosides, and diphosphatidylglycerol), the presence of 2-O-methyl-D-mannose, and their physiology (29). The first attempts to classify members of the genus Frankia were based on infectivity groups. Baker (4) grouped Frankia strains into the following four infectivity groups by using pure cultures in cross-inoculation tests: strains that infect Alnus and Myrica species, strains that infect Casuarina and Myrica species, strains that infect Elaeagnus and Myrica species, and strains that infect only Elaeagnus species. Lalonde et al. (28) used a more complex approach, in which they relied on diverse phenotypic characteristics, and described two species, Frankia alni and Frankia elaeagni. These attempts at classification did not include microsymbionts of members of known host genera from which isolates have not been obtained.

It has always been agreed that taxonomy should be consistent with phylogeny and that polyphyletic units, for instance, should not include clearly foreign taxa even though the foreign taxa may share several physiological characteristics with the polyphyletic units. This process has resulted in the splitting of some genera, such as the genera Pseudomonas and Rhizobium, and the lumping together of other taxa, such as Rhodococcus chlorophenolicus and the genus Mycobacterium (7). The phylogeny of the actinomycetes was recently reviewed by Embley and Stackebrandt (12), and these authors did not suggest that the status of the family Frankiaceae as one of the eight families of the order Actinomycetales should be modified. 16S rRNA and $16 \mathrm{~S}$ ribosomal DNA (rDNA) comparisons are becoming the preferred reference tools for bacterial taxonomy and phylogeny. This approach was used previously with partial (280nucleotide) sequences of a limited set of strains and yielded a 
TABLE 1. Bacteria used in this study

\begin{tabular}{|c|c|c|c|c|}
\hline $\begin{array}{l}\text { Trivial designation of strain } \\
\text { or organism }\end{array}$ & Characteristic or origin & Designation in trees & $\begin{array}{l}\text { Reference or } \\
\text { other designation }\end{array}$ & $\begin{array}{c}\text { GenBank } \\
\text { accession no. }\end{array}$ \\
\hline \multicolumn{5}{|l|}{ Frankia spp. strains } \\
\hline $\mathrm{ARgP5}$ & Alnus infective & FA-ARgP5 & $41 \mathrm{a}$ & L40612 \\
\hline $\mathrm{ACoN} 24 \mathrm{~d}$ & Alnus infective & FA-ACoN24d & 48 & L40610 \\
\hline AVN17s & Alnus infective & FA-AVN17s & 14 & L40613 \\
\hline $\mathrm{AgB} 1-9$ & Alnus infective & FA-AgB1-9 & 19 & L40611 \\
\hline Ea1-28 & Elaeagnus infective & FE-Ea12 & 14 & L40618 \\
\hline HR27-14 & Elaeagnus infective & FE-Hr27-14 & 14 & L40617 \\
\hline SCN10a & Elaeagnus infective & FE-SCN10a & $41 \mathrm{a}$ & L40619 \\
\hline PtI1 & Purshia tridentata isolate & F!-PtI1 & 4 & L41048 \\
\hline \multicolumn{5}{|l|}{ Geodermatophilus obscurus } \\
\hline subsp. obscurus & Amargosa Desert, Nev., soil & Geodermatophilus-obs-obscurus & ATCC 25078 & L40620 \\
\hline subsp. dictyosporus G97 & California soil & Geodermatophilus-obs-dictyosporus & 17 & L40621 \\
\hline \multicolumn{5}{|l|}{ Geodermatophilus obscurus } \\
\hline "Blastococcus aggregatus" & Baltic Sea & Blastococcus-aggregatus & ATCC 25902 & L40614 \\
\hline Dermatophilus congolensis & Scab from infected cattle & Dermatophilus-congolensis & ATCC 14637 & L40615 \\
\hline Actinoplanes sp. strain A8232 & Soil & Actinoplanes-sp. A8232 & This study & L41047 \\
\hline \multicolumn{5}{|l|}{ Unisolated nodule strains } \\
\hline Alnus rugosa $(\mathrm{Sp}+$ nodule $)$ & Trois-Rivières, Canada & AlnusR $^{a}$ & This study & L40956 \\
\hline Drvas drummondii & Gaspé, Canada & Dryas $^{a}$ & This study & L40616 \\
\hline Myrica nagi & Lawjinrew, Meghalaya, India & Myrica $^{a}$ & This study & L40622 \\
\hline
\end{tabular}

"Organism that was not isolated before molecular characterization.

preliminary Frankia phylogeny (39). The purpose of this study was to use complete $16 \mathrm{~S}$ rDNA sequences from a more comprehensive set of Frankia strains, avoiding strains having identical partial sequences (39), to obtain a more comprehensive view of the molecular phylogeny of this group of organisms.

\section{MATERIALS AND METHODS}

Bacterial strains. In this study we used eight Frankia strains, as well as unisolated nodular members of the genus Frankia and closely related actinomycetes (Table 1). The strains were grown at $28^{\circ} \mathrm{C}$ in $\mathrm{F}$ medium (Alnus-infective strains) (50), F medium containing Tween 80 (Elaeagnus-infective strains), or BAP medium (other strains) (38).

DNA extraction. Total DNAs were extracted from pure cultures (48) and from actinorhizal nodules (49) and used for amplification and sequencing.

PCR amplification. Double-stranded amplification was performed by using the whole rrs gene coding for 16S rRNA and a modification of the PCR procedure of Mullis and Faloona (37). The primers used to amplify the whole 16S rRNA gene that permitted subsequent cloning in a directional manner included primer FGPS5-255 (5'-TGGAAAGCTTGATCCTGGCT-3'), which contains a HindII restriction site, and primer FGPS1509'-153 (5'-AAGGAGGGGATCCAGCCG CA- $3^{\prime}$ ), which contains a Bam HI restriction site (the restriction sites are underlined). Each PCR was performed in a $50-\mu \mathrm{l}$ (final volume) reaction mixture containing template DNA, reaction buffer $(10 \mathrm{mM}$ Tris- $\mathrm{HCl}$ [pH 8.3$], 1.5 \mathrm{mM}$ $\mathrm{MgCl}_{2}, 50 \mu \mathrm{M} \mathrm{KCl}, 10 \%$ [wt/vol] gelatin), each deoxynucleoside triphosphate at a concentration of $200 \mathrm{mM}, 0.5 \mu \mathrm{M}$ oligonucleotides, and $2 \mathrm{U}$ of TaqI DNA polymerase (Gibco-BRL, Cergy-Pontoise, France). In some cases, the amplification reaction was performed directly with cells resuspended in amplification buffer. The amplification reactions were performed for 35 cycles, each of which consisted of denaturation at $95^{\circ} \mathrm{C}$ for $1 \mathrm{~min}$, annealing for $1 \mathrm{~min}$ at $55^{\circ} \mathrm{C}$, and extension at $72^{\circ} \mathrm{C}$ for $2 \mathrm{~min}$. To analyze the amplification products, $5 \mu \mathrm{l}$ of each reaction mixture was separated by electrophoresis on a $2 \%(\mathrm{wt} / \mathrm{vol})$ agarose gel (NuSieve; FMC, Rockland, Maine). The amplified material consisted of a 1,500-bp double-stranded DNA fragment. The amplified fragments were digested with BamHI and HindIII (digestion with HindIII had to be carried out overnight since the HindIII site is close to the extremities), cloned into Bam HI HindIII-cut pBluescript II SK- vector (Stratagene, La Jolla, Calif.), and transformed into Escherichia coli $\mathrm{DH} 5 \alpha \mathrm{F}^{\prime}$ (Bethesda Research Laboratories)

Sequencing of DNA fragments. Five or more clones were obtained, pooled, and used in the sequencing reaction to obscure possible errors due to the Taq polymerase or to differences between copies of the gene. Below, the designation of the primers used for sequencing refer to the "small-subunit ribosomal gene," the number in each designation is the coordinate of the 5 ' end in the Frankia sp. strain CeD homologous sequence (41) (GenBank accession number M55343), and a prime indicates that the primer is in the direction opposite transcription Primers FGPS310-20 (GAGACACGGCCCAGACTCCT), FGPS485-292 (CAG CAGCCGCGGTAA), FGPS747-293 (AACAGGATTAGATAC), FGPS1047295 (ATGTTGGGTTAAGTC), FGPS1156-39 (GACGTCAAGTCACATGC
CC) FGPS305'-78 (CCAGTGTGGCCGGTCGCCCTCTC), FGPS505'-313 (GTATTACCGCGGCTGCTG), FGPS659'64 (CACCGCTACACCAGGAAT TC), FGPS $910^{\prime}-270$ (AGCCTTGCGGCCGTACTCCC), and FGPS1176'-112 (GGGGCATGATCACTTGACGTC), as well as primers T3 and T7 (Pharmacia), were necessary to determine the sequence of the whole 16S rDNA molecule for both strands. The dideoxy chain termination sequencing procedure was performed by using T7 sequencing kits (Pharmacia LKB, Uppsala, Sweden). The sequence of Frankia sp. strain PtI1 was determined as described previously (20).

Data analysis. GenBank release 86 was scanned for related sequences by using the algorithms FASTA of Lipman and Pearson (31) as implemented by Higgins and Gouy (21) and BLAST (3). Sequences were aligned by using CLUSTAL V (22). Indel-containing regions (regions containing an insertion in one sequence or a deletion in another sequence) were excluded from the analysis. Matrix pairwise comparisons were corrected for multiple-base substitutions by the method of Kimura (25). Phylogenetic trees were constructed by the neighborjoining $(46)$ and parsimony $(11,26)$ methods. A bootstrap confidence analysis was performed with 1,000 replicates to determine the reliability of the distance tree topologies obtained (13). The resulting tree was drawn by using the NJPlot software of M. Gouy (Laboratoire de Biométrie, U.R.A. C.N.R.S. 2055, Université Lyon I).

\section{RESULTS}

Amplification from chromosomal DNA. Chromosomal DNAs of Frankia spp. that were obtained from pure cultures or nodules were used as sources of amplifiable material. In all cases, the amplified DNA was a single product of the expected size (approximately 1,500 bp between primers FGPS5-255 and FGPS1509'-153). Several clones (more than five) were pooled to avoid errors due to Taq amplification.

Sequencing and comparison of amplified $16 \mathrm{~S}$ rDNAs. We determined the complete $16 \mathrm{~S}$ rDNA sequences of all of the strains studied (Fig. 1) except the amplified fragment from Actinoplanes sp. isolate A8232, which has a HindIII site at coordinate 180 . The sequence of this uncloned fragment had to be determined later by direct sequencing (39). The sequences were aligned with previously published $16 \mathrm{~S}$ rRNA sequences of Frankia strains available from the GenBank database (Table 2).

In some cases amplification of nodule DNAs with the primers which we used resulted in amplification of Frankia-like sequences, as in the case of Dryas drummondii. In other cases mostly chloroplast-like sequences were obtained (for example, with Alnus crispa). A search of the GenBank database (by 


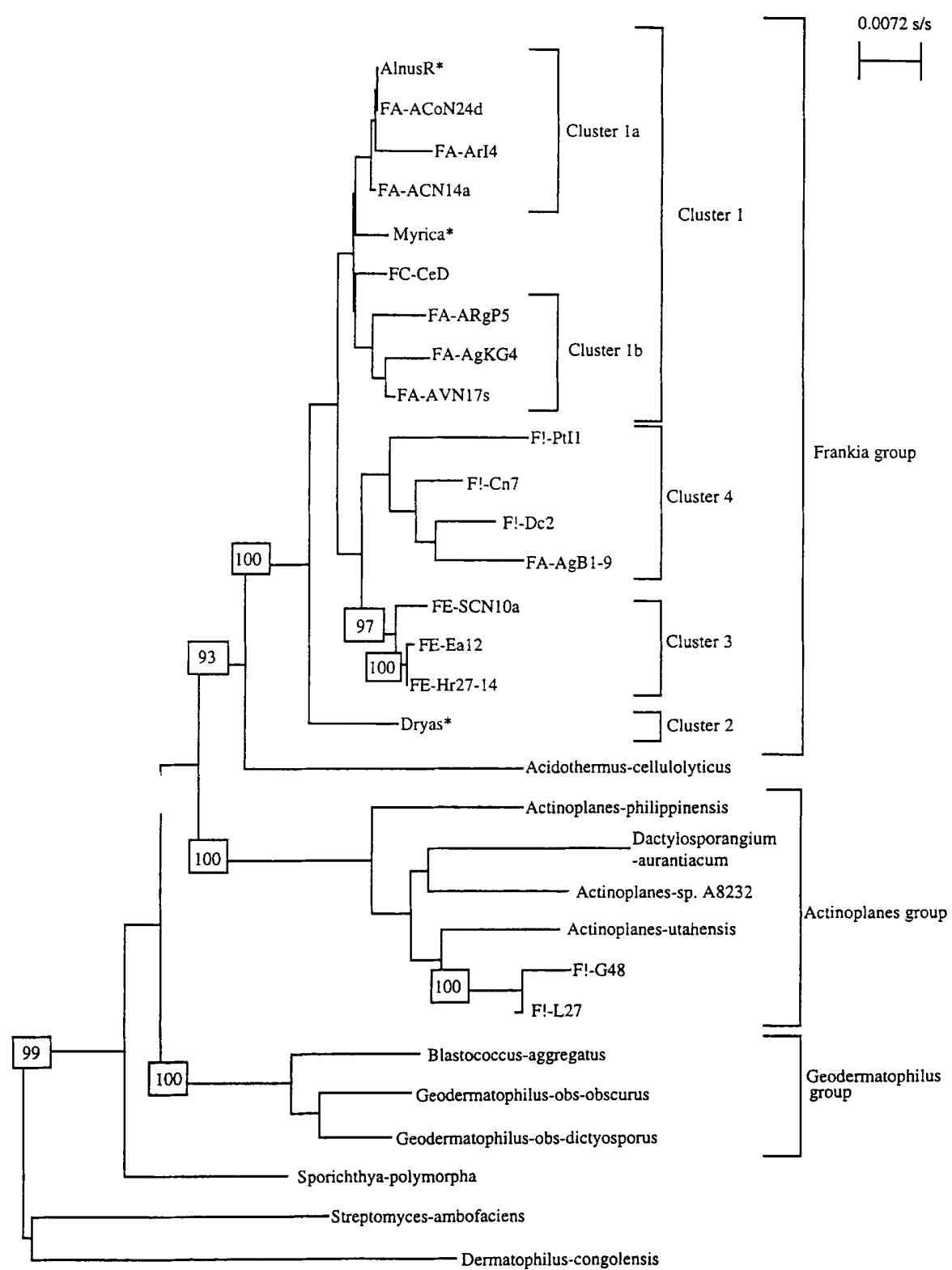

FIG. 1. Phylogenetic tree obtained by the neighbor-joining method (46). The Streptomyces ambofaciens and Dermatophilus congolensis sequences were used to define the root. The numbers in the boxes are bootstrap values; only the values that were greater than $90 \%$ are shown. A total of 1,389 sites were used for the calculations. Scale bar $=0.0072$ substitution per site $(\mathrm{s} / \mathrm{s})$. An asterisk indicates that the Frankia strain was not isolated prior to molecular characterization.

using the BLAST or FASTA algorithms) revealed that the closest relatives of the Alnus crispa $16 \mathrm{~S}$ rRNA amplicon were Nicotiana plumbaginifolia (locus TOBCP16SRD) and Alnus incana (locus AIU03555. RR1) chloroplast 16S rRNAs. In the case of Alnus rugosa nodules, 5 of 10 clones had chloroplastlike sequences, while the other 5 clones had typical Frankia sequences, which were included in this study. A comparison of the chloroplast $16 \mathrm{~S}$ rRNA sequences with other bacterial $16 \mathrm{~S}$ rRNA sequences revealed that a $\mathrm{T}$ was present in most chloroplast sequences at coordinate 30 , while a $C$ was present in all other bacteria, including, surprisingly, all cyanobacteria. This finding allowed us to synthesize primer FGP4-330 (5'-ATGGA GAGTTTGATCATGGCTCAGGAC-3'), which contained a $B c l$ restriction site (underlined) (used for cloning) and effectively amplified nonchloroplast $16 \mathrm{~S}$ rDNA preferentially from plant tissues. The addition to the amplification reaction mix- ture of restriction enzyme $\mathrm{NruI}$, for which a site is present in most chloroplast $16 \mathrm{~S}$ rRNAs but absent in actinomycetes rRNAs, also helped eliminate unwanted chloroplast 16S rRNA molecules.

Clustering. Table 3 shows the numbers of substitutions for Frankia strains and related strains when regions of ambiguous alignment were not considered. The following clusters were identified by using the distance matrix and the resulting phylogenetic tree (Fig. 1).

Cluster 1 is a very broad group that contains the strains that infect Alnus species. Strain ACoN24d, which belongs to the genomic species $F$. alni (subcluster 1a), is very closely related to the unisolated strain found in Alnus Sp+ nodules; we identified only a few substitutions in indel-containing regions, which were not considered in the analysis. These organisms form a tight cluster with strains ArI4 and ACN14a. Strain ArI4 
TABLE 2. 16S rRNA sequences obtained from GenBank

\begin{tabular}{llll}
\hline \multicolumn{1}{c}{ Organism } & \multicolumn{1}{c}{ Source } & $\begin{array}{c}\text { GenBank } \\
\text { accession no. }\end{array}$ & \multicolumn{1}{c}{ Designation in trees } \\
\hline Frankia sp. strains & & & \\
ACN14a & Alnus crispa, Canada & M88466 & FA-ACN14a \\
ArI4 & Alnus rugosa, United States & L11307 & FA-ArI4 \\
AgKG4-84 & Alnus glutinosa, The Netherlands & L18976 & FA-AgKG4 \\
CeD & Casuarina equisetifolia, Senegal & M55343 & FC-CeD \\
L27 & Podocarpus sp., People's Republic of China & L11306 & F!-L27 \\
G48 & Podocarpus sp., People's Republic of China & M59075 & F!-G48 \\
Cn7 & Coriaria nepalensis, Pakistan & L18982 & F!-Cn7 \\
Dc2 & Datisca cannabina, Pakistan & L18978 & F!-Dc2 \\
Acidothermus cellulolyticus & & X70635 & Acidothermus-cellulolyticus \\
Actinoplanes philippinensis & & X72864 & Actinoplanes-philippinensis \\
Actinoplanes utahensis & & X80823 & Actinoplanes-utahensis \\
Dactylosporangium aurantiacum & & X72779 & Dactylosporangium-aurantiacum \\
Sporichthya polymorpha & & X72377 & Sporichthya-polymorpha \\
Streptomyces ambofaciens & & M27245 & Streptomyces-ambofaciens \\
\hline
\end{tabular}

is the most distantly related member of this group; it also has the sequence that contains the highest number of undetermined positions and presumably also the highest number of errors. For this reason, strain ArI4 was always only distantly related to the other subcluster 1a organisms. Closely related to this subcluster are Casuarina-infective Frankia sp. strain CeD and the unisolated Frankia microsymbiont present in Myrica nagi. The other Alnus-infective strains, which form subcluster $1 \mathrm{~b}$, may or may not cluster with the members of subcluster $1 \mathrm{a}$ depending on which regions are considered. A parsimony analysis also revealed subcluster 1 a containing $F$. alni (as well as the Alnus $\mathrm{Sp}+$ nodule strain and strains ArI4, ACN14a, and ACoN24d), but not subcluster 1 b or cluster 1 (data not shown).

In cluster 2 the closest neighbors of the Dryas microsymbiont are the two unisolated microsymbionts present in the root nodules of Coriaria nepalensis and Datisca cannabina. These three organisms form a tight and very coherent group (data not shown). The Dryas microsymbiont sequence was the only sequence used for further analysis since the other sequences were not complete and were very similar to the sequence of the Dryas microsymbiont. The Dryas microsymbiont had no close neighbors on the neighbor-joining tree, while it was closely related to the Casuarina-infective strain on the parsimony tree.

The three Elaeagnus-infective strains form a coherent cluster 3 (97\% of the bootstrap replicates). In this cluster the region where the level of sequence divergence is highest is the region between coordinates 900 and 1200 , which has been described for 10 strains (39). Because there is less variability at coordinates 1 to 300 (unpublished data), we decided not to determine complete sequences for all of the Elaeagnus-infective strains. This cluster was also detected by parsimony analysis (data not shown). The sequences of the three cluster 3 strains exhibited only nine mismatches, one in the first, fifth (coordinate 180) and eight between coordinates 974 and 1267.

The three atypical (noninfective and non-nitrogen-fixing) strains obtained from Coriaria, Datisca, and Purshia species form a broad group designated cluster 4 (bootstrap value, 77\%) together with Alnus-infective, non-nitrogen-fixing strain AgB1-9. This group was not detected by parsimony analysis.

The four clusters described above form a highly coherent Frankia group (with a bootstrap value of $100 \%$ ). This group was also identified by parsimony analysis, but the parsimony analysis group differed in that it included the genus Acidothermus.

In the Actinoplanes group, which is farther away from the
Frankia group, are the so-called Frankia strains isolated from Podocarpus macrophylla (strain L27) and Podocarpus nagi (strain G48) in Fujian Province, People's Republic of China, by D. C. Yang, Y. Shi, and J. S. Ruan (29a), and these organisms form a relatively tight cluster together with Actinoplanes sp. strain A8232, which was identified on the basis of its morphology (multilobed sporangia, motile spores arranged in coils) and biochemistry (type II cell wall containing meso-diaminopimelic acid, arabinose, xylose, and glycine and type PII phospholipids, including phosphatidylethanolamine). This group was well supported by a bootstrap value of $100 \%$ and by the results of the parsimony analysis.

In the Geodermatophilus group the Geodermatophilus and "Blastococcus" sequences form a tight and coherent group ( $100 \%$ of the bootstrap replicates), and this group was also identified by parsimony analysis.

We found that two other sequences, the sequences of Sporichthya polymorpha and Acidothermus cellulolyticus, clustered with the Frankia and Geodermatophilus sequences. Acidothermus cellulolyticus clustered with the Frankia group in $93 \%$ of the bootstrap replicates, and in the parsimony analysis, this organism also fell in the Frankia group.

The Frankia, Actinoplanes, and Geodermatophilus groups, together with Acidothermus cellulolyticus and S. polymorpha, form a coherent ( $99 \%$ of bootstrap replicates) subline of descent within the Actinomycetales.

The Dermatophilus congolensis sequence was found to be only distantly related to the other sequences in all analyses.

\section{DISCUSSION}

The phylogeny of the genus Frankia and related taxa was analyzed by examining a distance matrix and performing a parsimony analysis with the $16 \mathrm{~S}$ rDNA sequences. The distance matrix (Table 3 ) and a phylogenetic tree (Fig. 1) were constructed by transforming variations in the 16S rDNA sequences into evolutionary distance values. Our findings illustrate the relationships among Frankia spp. strains, as well as the relationships between these strains and unclassified strains. The following four main clusters were revealed by this phylogenetic analysis: (i) $F$. alni, the closely related Casuarina-infective Frankia strain, and a second subcluster of Alnus-infective strains; (ii) unisolated microsymbionts of Dryas, Coriaria, and Datisca species; (iii) the Elaeagnus-infective Frankia strains; and (iv) the atypical Frankia isolates (including infective but 


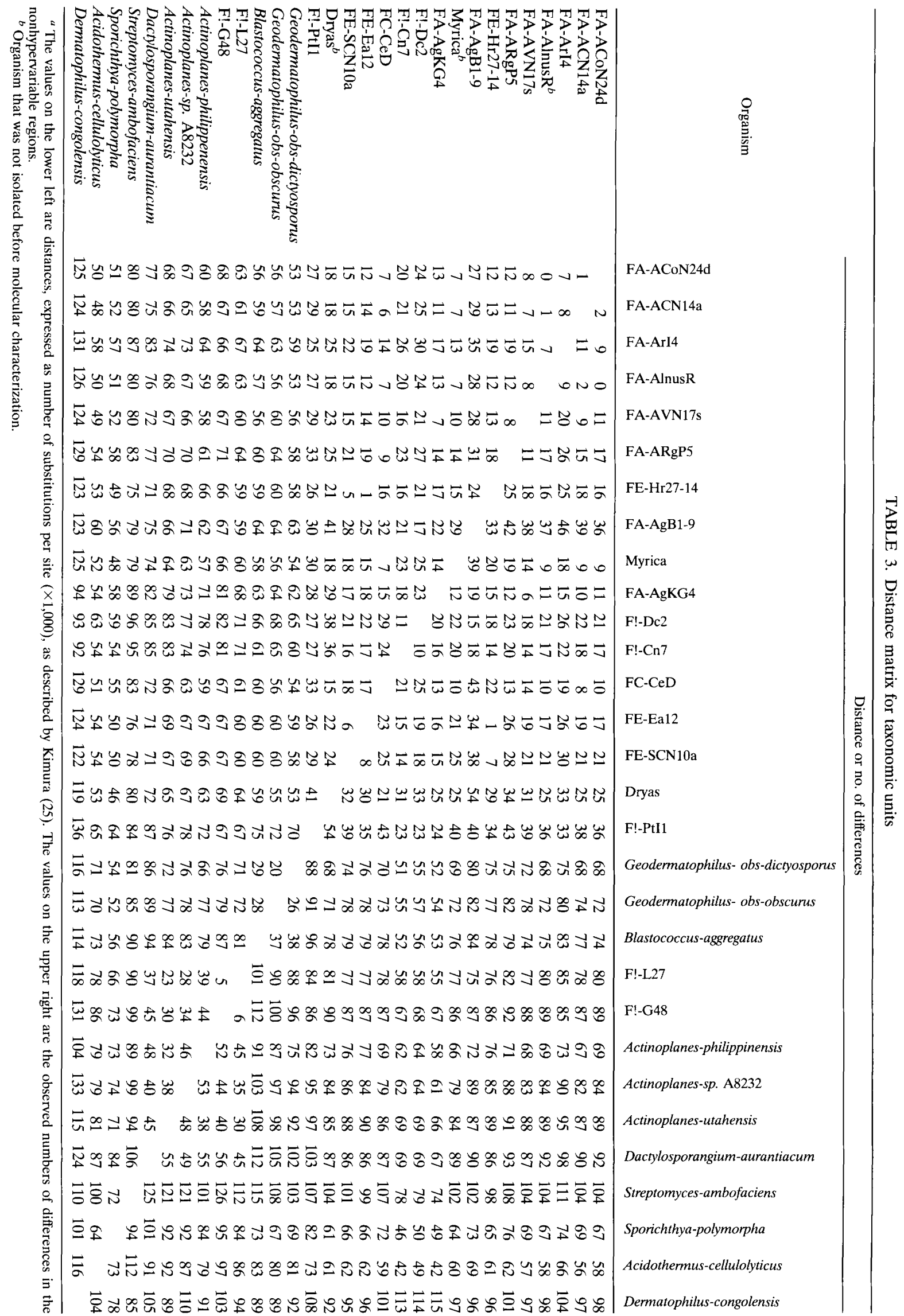


non-nitrogen-fixing strain AgB1-9). The four clusters were coherent and together formed the Frankia group. As determined in this analysis, the Alnus-infective strains (cluster 1) are by far the most diverse strains (largest difference not including indelcontaining regions, $1.9 \%$ ). The Elaeagnus-infective group (cluster 3) exhibited far less diversity (nine mismatches overall or $0.6 \%$ difference between strain SCN10a and strain Ea1-12). These organisms were predicted by the results of a PCR restriction fragment length polymorphism analysis of the nifDK intergene sequence (23) to be among the most distant isolates in that group of strains. Authors who arrived at different conclusions $(16,28)$ used a set of closely related Alnus-infective strains that were characteristic of the strains consistently isolated in America as well as in Europe.

(i) $F$. alni and the $\mathbf{S p}+$ phenotype (subcluster 1a). Our study included an unisolated Frankia strain obtained from a Alnus rugosa nodule in Canada. Using the PCR technique, we were able to specifically amplify the $16 \mathrm{~S}$ rDNA of this strain by using total DNA extracted from the Alnus nodule. This strain corresponds to a phenotype designated $\mathrm{Sp}+(52)$, which is rarely isolated in pure culture, and grouped very closely with $F$. alni, as did another Alnus crispa $\mathrm{Sp}+$ nodule strain obtained from the French Alps (39). Thus, we concluded that a major subdivision of the genus Frankia should not be created for the Sp+ phenotype (28).

(ii) Subcluster 1b: the second Alnus-infective group of strains. The second group of Alnus-infective strains (which does not include $A$ lnus-infective, non-nitrogen-fixing strain AgB1-9) (19) is made up of at least three strains, two of which belong to different genomic species (14). This group is not well defined, as indicated by the differences in topology observed with the method which we used, and the data probably reflect the large distances that exist between the strains. The fossil record suggests that the genera Alnus and Myrica are the oldest actinorhizal genera $(33,51)$. Cross-infectivity studies (4) have shown that the genus Myrica is a promiscuous host and thus has the largest potential range of infective strains. In the case of the genus Alnus, studies have revealed that the actual diversity of strains that are isolated from and infect Alnus species is great. The deep branching of strains that infect Myrica and Alnus species and the ancient appearance of Alnus and Myrica species in the fossil record suggest that the other groups of Frankia strains may have evolved from the cluster 1 strains as other host plants for Frankia strains evolved.

Organisms related to subclusters $1 \mathrm{a}$ and $1 \mathrm{~b}$ include Casuarina-infective strain CeD and the Myrica nagi microsymbiont. The relative positions of the four subclusters are impossible to locate precisely with the data available.

(iii) Dryas microsymbionts. The unisolated microsymbiont present in Dryas species in Canada (where neither Coriaria nor Datisca species grow) was found to be very closely related to the other unisolated microsymbionts present in Coriaria and Datisca species in Pakistan (34), Mexico, New Zealand, and France (40). No strains in this well-defined taxon (100\% of bootstrap replicates) have been induced to grow in the media used for pure-culture isolation of Frankia strains. On the other hand, morphological features (host specificity of crushed nodules, diameter of hyphae, size and shape of nitrogen-fixing vesicles) of the Frankia strains in nodules led Becking (5) to consider two of these three microsymbionts members of different species. The third microsymbiont infected Datisca plants that were not then known to be actinorhizal; these plants were later discovered to be nodulated by Frankia strains (9) and to have a unique morphology inside their nodules (round diazovesicles oriented toward the center of the host cell, with an excentric stele). The level of similarity of the sequences of these three organisms is high, and two of these organisms (the Coriaria and Datisca microsymbionts) can be cross-inoculated (34). On the other hand, it has been determined by $r b c L$ sequence analysis that the Rosaceae (which includes the genus Dryas) is quite distant from the Datiscaceae and the Coriariaceae $(2,10)$. It is likely that Frankia morphology within nodules is determined by the host plant, as has been shown to be the case in Alnus and Myrica species (27). In spite of their different host plants the three microsymbionts form a welldefined and statistically significant cluster.

(iv) Elaeagnus-infective strains. The three Elaeagnus-infective Frankia strains, which form well-defined cluster 2, have most of their mismatches between coordinates 974 and 1267. This is in contrast to most other pairs of sequences (for example, the ACoN24d and ACN14a sequences), in which most mismatches are between coordinates 83 and 182; this conforms to the general pattern of the $16 \mathrm{~S}$ rRNA molecule (54). For this reason the complete $16 \mathrm{~S}$ rRNA sequences of other Elaeagnusinfective strains were not determined, as partial sequencing of coordinates 904 to 1188 (39) and coordinates 1 to 260 (47) revealed that different Elaeagnus-infective genomic species had at most nine mismatches.

(v) Atypical strains. Unclassified strain PtI1, which was isolated from Purshia tridentata, was the only isolate obtained from a rosaceous plant. The family Rosaceae comprises several nonactinorhizal taxa of economic importance, such as raspberry and strawberry plants. Our phylogenetic tree (Fig. 1) revealed that strain PtI1 is separated from the other Frankia strains and genomic species, which is consistent with the specific characteristics of this strain. Strain PtI1 was reported to be noninfective on its original host plant and not to hybridize with nif probes (39). Its closest relatives are atypical strains $\mathrm{Cn} 7$ and Dc2, which were isolated from Coriaria and Datisca species, respectively (35), and which share with PtI1 the inability to reinfect their original hosts and also lack detectable nif genes. These strains group with strain AgB1-9, an Alnus-infective strain, with which they share an inability to fix nitrogen and a lack of nif hybridizing fragments (19). This broad cluster was not significant as determined by a bootstrap analysis and was not found in the parsimony study.

Family Frankiaceae. Grouping the Geodermatophilus strains with the genus Frankia, as proposed by Fox and Stackebrandt (15) and Hahn et al. (18), is problematic. Both the distance matrix analysis and the parsimony analysis results group the Actinoplanes cluster with the genus Frankia but not with the coherent Geodermatophilus-"Blastococcus" group. These clustering results can change according to the number of sequences, the strains included in the study, and the regions considered (because of the presence of indel-containing regions). The findings to date suggest that the three groups are equidistant from each other.

The results described above indicate that the phylogenetic tree derived from an analysis of complete $16 \mathrm{~S}$ rDNA sequences is different from the tree based on 16S rRNA oligonucleotide catalogs and partial sequences (18); the Actinoplanes group is at least as close to the genus Frankia as the Geodermatophilus-"Blastococcus" group is.

Even if the average catalog $\left(S_{\mathrm{AB}}\right)$ values for Actinoplanes and Frankia strains could be considered high enough $\left(S_{\mathrm{AB}}\right.$, 0.51 to 0.55 ) (12), the relationships found when $16 \mathrm{~S}$ rRNA oligonucleotide catalogs were used were not confirmed by the results of a study of the entire $16 \mathrm{~S}$ rDNA molecule. Because of the imprecise relationship between $S_{\mathrm{AB}}$ and sequence similarity (54), catalog $S_{\mathrm{AB}}$ values are used only for preliminary clustering, while the use of these values in cladistic analyses can lead to erroneous conclusions, as shown by the results of this 


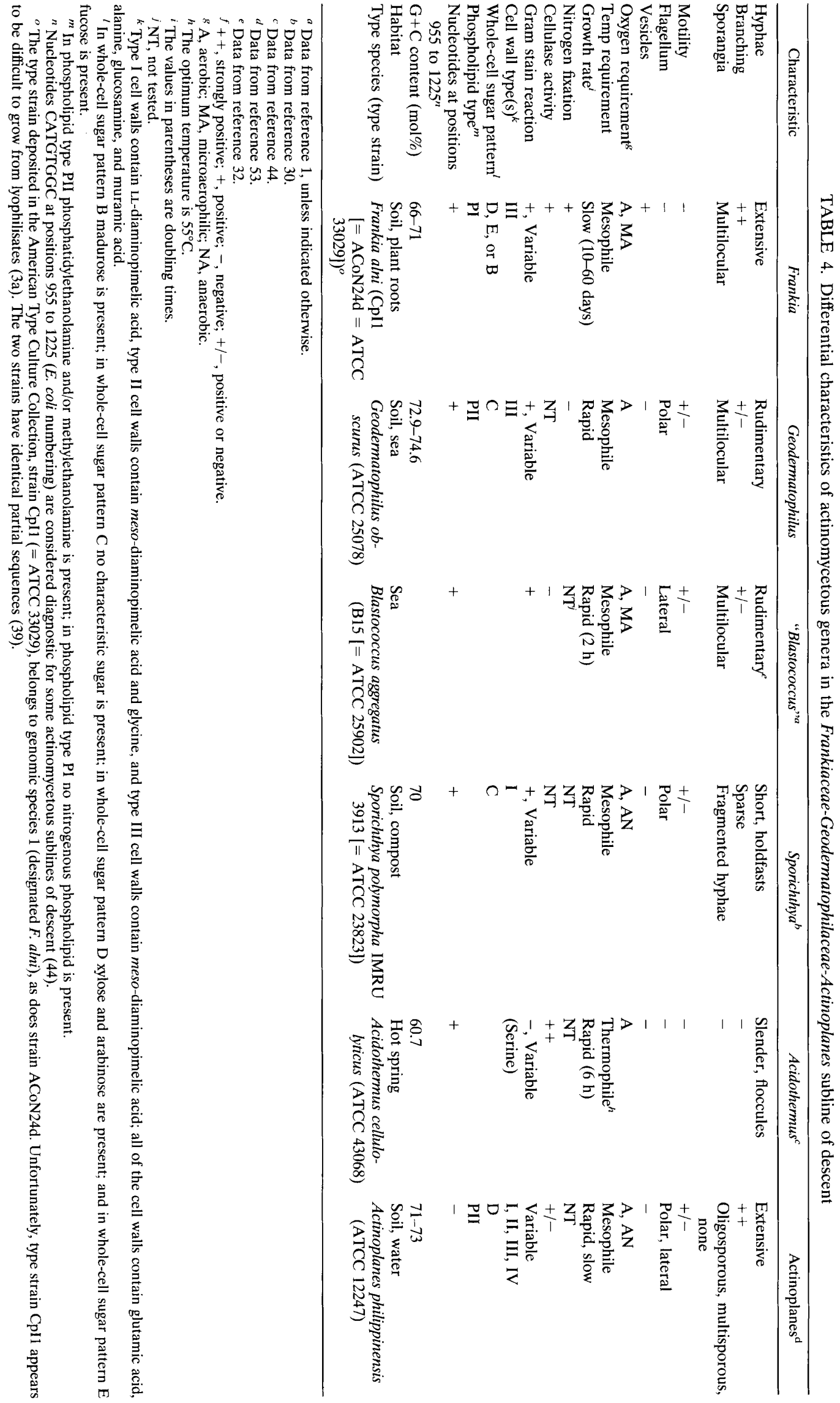


study based on 16S rDNA sequence comparisons, a more precise technique.

One possible result is to place the Geodermatophilus group in the family Frankiaceae, in which case the genus Actinoplanes would also belong to this family together with the thermophilic cellulolytic nonsporulating rod-shaped organism Acidothermus cellulolyticus (44) and maybe also the compost-inhabiting motile spore-forming organism S. polymorpha (45), thus ending their "search for a family" (30). Another possible result is to consider the family Actinoplanaceae and the Geodermatophilus"Blastococcus" group two distinct families that are closely related to the Frankiaceae. When we considered phenotypic data, which must be consistent with phylogenetic results, the genera Geodermatophilus and Frankia had few distinctive characteristics compared with other actinomycetes other than multilocular sporangia. The cell wall type (type III) cannot be considered a reliable criterion as many morphologically, physiologically, and ecologically different actinomycetes have this cell wall type. On the other hand, several phenotypic features are shared by the genera Geodermatophilus and Actinoplanes, including spore motility, phospholipid type PII, peptidoglycan type $A 1 \gamma$, and major menaquinone MK- $9\left(\mathrm{H}_{4}\right)$.

Since the first possibility would result in lumping together taxa that have clearly different taxonomic properties (Table 4), we propose that the Geodermatophilus-"Blastococcus" group should be placed in a family of its own (the family Geodermatophilaceae) and that the paraphyletic family Frankiaceae as defined by Hahn et al. (18) should be emended.

Time scale of Frankia evolution. According to Ochman and Wilson (42) and Moran et al. (36), the evolutionary rate for the whole $16 \mathrm{~S}$ rRNA molecule between pairs of operational taxonomic units was 1 to $2 \%$ per $50 \times 10^{6}$ to $55 \times 10^{6}$ years during the last $500 \times 10^{6}$ years. The maximum distance for the large coherent group containing the four Frankia lineages is about $4 \%$, which corresponds to about $100 \times 10^{6}$ to $200 \times 10^{6}$ million years; this value is consistent with the time of appearance of most major groups of angiosperms, including the Myricaceae and Betulaceae, which appeared $90 \times 10^{6}$ to $100 \times 10^{6}$ years ago (33). The other Frankia lineages are more similar to one another; for instance, the distance between the Elaeagnusinfective Frankia strains and the $F$. alni lineage is $1 \%$, which corresponds to about $25 \times 10^{6}$ to $50 \times 10^{6}$ years. This value is consistent with the time of appearance of the Elaeagnaceae, estimated to be about $30 \times 10^{6}$ years ago on the basis of the fossil record. On the other hand, the Casuarina-infective strains and the $F$. alni lineage differ by $0.4 \%$, which corresponds to a time of appearance of $10 \times 10^{6}$ to $20 \times 10^{6}$ years ago, well after the first occurrence of Casuarina pollen (about $80 \times 10^{6}$ years ago) (24). It is noteworthy that these estimates of evolutionary timing concur, but imprecise dating methods preclude firm conclusions.

In this study we found that we could characterize and classify strains in pure culture and that we could also characterize at the molecular level new strains that are present in nodule tissue without the difficult and time-consuming task of isolating the symbionts from nodules. By obtaining genetic information in this manner for undescribed Frankia strains, such as the symbionts of actinorhizal hosts belonging to the Coriariaceae, Datiscaceae, Rhamnaceae, or Rosaceae, it should be possible to determine the relationships of these organisms with wellknown strains isolated from Alnus, Elaeagnus, or Casuarina root nodules.

Emendation of the family Frankiaceae Hahn et al. 1989. Hyphae are extensive. Aerial mycelium is absent. The hyphal diameter varies from 0.5 to $2.0 \mu \mathrm{m}$. Branching is limited to extensive. The mycelium may bear terminal or lateral thick- walled diazovesicles. A part of the thallus is composed of irregularly shaped cuboid to oval cells that divide in more than one plane. These cells are nonmotile, possess an outer membrane, and may germinate to give filaments. Gram positive to gram variable. Aerobic to microaerophilic. The nitrogen sources used are amino acids and ammonia; atmospheric nitrogen is fixed in vivo and in vitro by most strains. The carbon sources used include carbohydrates, organic acids, and fatty acids. Found in soil and as symbionts of higher plants. The cell wall type is type III (meso-diaminopimelic acid, glutamic acid, alanine, glucosamine, and muramic acid). The whole-cell sugar patterns include patterns B (3-O-methyl-D-galactose, madurose), D (xylose), and $\mathrm{E}$ (fucose). 2-O-Methyl-D-mannose is a diagnostic sugar. Phospholipid pattern PI. The major menaquinone is MK-9 $\left(\mathrm{H}_{4}\right)$. Fatty acid pattern 1 . All strains tested contain hopanoid lipids (25a). The family comprises the single genus Frankia.

Emendation of the family Geodermatophilaceae. Hyphae are rudimentary. Aerial mycelium is absent. The hyphal diameter varies from 0.5 to $2.0 \mu \mathrm{m}$. The thallus is composed of irregularly shaped cuboid to oval cells that divide in more than one plane. These cells serve as propagules, may be motile or nonmotile, and may germinate to produce filaments or buds, which develop into motile cells. Gram positive. Aerobic. Members utilize sources of nitrogen such as amino acids and ammonia and do not fix nitrogen. The carbon sources used include carbohydrates, organic acids, and fatty acids. Found in soil and in the sea. The cell wall type is type III. The whole-cell sugar pattern is pattern $\mathrm{C}$ (no characteristic sugars). Phospholipid pattern PII. The major menaquinone is MK- $9\left(\mathrm{H}_{4}\right)$. Fatty acid pattern $2 b$. Peptidoglycan type A1 $\gamma$. This family comprises the genus "Blastococcus" and the genus Geodermatophilus.

\section{ACKNOWLEDGMENTS}

Philippe Normand's work was supported by IFCPAR grant A603-1. We thank M. P. Lechevalier (Waksman Institute of Microbiology, Rutgers, N.J.), Neil McEwan (University of Glasgow, Glasgow, United Kingdom), and D. Hahn (ETH, Zurich, Switzerland) for helpful comments and M. S. Mirza (Islamabad, Pakistan) for providing strain AgB1-9.

\section{REFERENCES}

1. Ahrens, R., and G. Moll. 1970. Ein neues knospendes Bakterium aus der Ostsee. Arch. Mikrobiol. 70:243-265.

2. Albert, V. A., M. W. Chase, and S. E. Williams. 1992. Carnivorous plants: phylogeny and structural evolution. Science 257:1491-1495

3. Altschul, S. F., W. Gish, W. Miller, E. W. Myers, and D. J. Lipman. 1990. Basic local alignment search tool. J. Mol. Biol. 215:403-410.

3a.American Type Culture Collection. Personal communication.

4. Baker, D. D. 1987. Relationships among pure cultured strains of Frankia based on host-specificity. Physiol. Plant. 70:245-248.

5. Becking, J. H. 1970. Frankiaceae, fam. nov. (Actinomycetales) with one new combination and six new species of the genus Frankia Brunchorst 1886, 174. Int. J. Syst. Bacteriol. 20:201-220.

6. Benson, D. R., and W. B. Silvester. 1993. Biology of Frankia strains, actinomycete symbionts of actinorhizal plants. Microbiol. Rev. 57:293-319.

7. Briglia, M., R. I. L. Eggen, D. J. Van Elsas, and W. M. De Vos. 1994. Phylogenetic evidence for transfer of pentachlorophenol-mineralizing Rhodococcus chlorophenolicus PCP-I ${ }^{\mathbf{T}}$ to the genus Mycobacterium. Int. J. Syst. Bacteriol. 44:494-498.

8. Callaham, D., P. Del Tridici, and J. G. Torrey. 1978. Isolation and cultivation in vitro of the actinomycete causing root nodulation in Comptonia. Science 199:899-902.

9. Calvert, H. E., A. H. Chaudhary, and M. Lalonde. 1979. Structure of an unusual root nodule symbiosis in a non-leguminous herbaceous dicotyledon, p. 474-475. In J. C. Gordon, C. T. Wheeler, and D. A. Perry (ed.), Symbiotic nitrogen fixation in the management of temperate forests. Forest Research Laboratory, Oregon State University, Corvallis.

10. Chase, M. W., D. E. Soltis, R. G. Olmstead, D. Morgan, D. H. Les, B. D. Mishler, M. R. Duvall, R. A. Price, H. G. Hills, Y.-L. Oiu, K. A. Kron, J. H. Rettig, E. Conti, J. D. Palmer, J. R. Manhart, K. J. Sytsma, H. J. Michaels, W. J. Kress, K. G. Karol, W. D. Clark, M. Hedroen, B. S. Gaut, R. K. Jansen, 
K.-J. Kim, C. F. Wimpee, J. F. Smith, G, R, Furnier, S. H. Strauss, Q.-Y. Xiang, G. M. Plunkett, P. S. Soltis, S. Swensen, S. E. Williams, P. A. Gadek, C. J. Quinn, L. E. Eguiarte, E. Golenberg, G. H. Learn, Jr., S. W. Graham, S. C. Barrett, S. Dayanandan, and V. A. Albert. 1993. DNA sequence phylogenetics of seed plants: an analysis of the plastid gene $r b c L$. Ann. Mo. Bot. Gard. 80:528-580.

11. Eck, R. V., and M. O. Dayhoff. 1966. Atlas of protein sequence and structure. National Biomedical Research Foundation, Silver Spring, Md

12. Embley, T. M., and E. Stackebrandt. 1994. The molecular phylogeny and systematics of the actinomycetes. Annu. Rev. Microbiol. 48:257-289.

13. Felsenstein, J. 1985. Confidence limits on phylogenies: an approach using the bootstrap. Evolution 39:783-791.

14. Fernandez, M. P., H. Meugnier, P. A. D. Grimont, and R. Bardin. 1989 Deoxyribonucleic acid relatedness among members of the genus Frankia. Int. J. Syst. Bacteriol. 39:424-429.

15. Fox, G. E., and E. Stackebrandt. 1976. The application of 16S rRNA cataloguing and 5S rRNA sequencing in microbial systematics, p. 405-458. In R. R. Colwell and R. Grigorova (ed.), Methods in microbiology. Academic Press, Orlando, Fla.

16. Gardes, M., J. Bousquet, and M. Lalonde. 1987. Isozyme variation among 40 Frankia strains. Appl. Environ. Microbiol. 53:1596-1603.

17. Goodfellow, M., and T. Pirouz. 1982. Numerical classification of sporoactinomycetes containing meso-diaminopimelic acid in the cell wall. J. Gen. Microbiol. 128:503-527.

18. Hahn, D., M. P. Lechevalier, A. Fischer, and E. Stackebrandt. 1989. Evi dence for a close phylogenetic relationship between members of the gener Frankia, Geodermatophilus, and "Blastococcus" and emendation of the family Frankiaceae. Syst. Appl. Microbiol. 11:236-242.

19. Hahn, D., M. J. C. Starrenburg, and A. D. L. Akkermans. 1988. Variable compatibility of cloned Alnus lutinosa ecotypes against ineffective Frankia strains. Plant Soil 107:233-243.

20. Harry, D. E., D. C. Yang, and J. O. Dawson. 1991. Nucleotide sequence and diversity in 16S ribosomal RNA from Frankia. Plant Soil 131:143-146.

21. Higgins, D., and M. Gouy. 1987. Interfacing similarity search software with the sequence retrieval system ACNUC. Comput. Applic. Biosci. 3:239 241 .

22. Higgins, D. G., and P. M. Sharp. 1988. CLUSTAL: a package for performing multiple sequence alignments on a microcomputer. Gene 73:237-244.

23. Jamann, S., M. P. Fernandez, and P. Normand. 1993. Typing method for $\mathrm{N}_{2}$-fixing bacteria based on PCR/RFLP-application to the characterization of Frankia strains. Mol. Ecol. 2:17-26.

24. Johnson, L. A. S., and K. L. Wilson. 1989. Casuarinaceae: a synopsis. Syst. Assoc. Spec. Vol. 40B:87-116.

25. Kimura, M. 1980. A simple method for estimating evolutionary rates of base substitutions through comparative studies of nucleotide sequences. J. Mol Evol. 16:111-120.

25a.Kleemann, G., D. D. Baker, and A. Berry. Personal communication

26. Kluge, A. G., and J. S. Farris. 1969. Quantitative phyletics and the evolution of anurans. Syst. Zool. 18:1-32.

27. Lalonde, M. 1979. Immunological and ultrastructural demonstration of nodulation of the European Alnus glutinosa (L.) Gaertn. host plant by an actinomycetal isolate from the North American Comptonia peregrina (L.) Coult. root nodule. Bot. Gaz. Suppl. 140S:\$35-S43.

28. Lalonde, M., L. Simon, J. Bousquet, and A. Séguin. 1988. Advances in the taxonomy of Frankia: recognition of species alni and eleagni and novel subspecies pommerii and vandijkii, p. 671-680. In H. Bothe, F. J. de Bruijn, and W. E. Newton (ed.), Nitrogen fixation: hundred years after. Gustav Fischer Verlag, Stuttgart, Germany.

29. Lechevalier, M. P. 1994. Taxonomy of the genus Frankia (Actinomycetales) Int. J. Syst. Bacteriol. 44:1-8

29a.Lechevalier, M. P. Personal communication.

30. Lechevalier, M. P., H. A. Lechevalier, and P. E. Holbert. 1968. Sporichthya, un nouveau genre de Streptomycetaceae. Ann. Inst. Pasteur (Paris) 114:277-286.

31. Lipman, D. J., and W. R. Pearson. 1985. Rapid and sensitive protein similarity searches. Science 227:1435-1441.

32. Luedemann, G. M., and A. F. Fonseca. 1989. Genus Geodermatophilus Lu- edemann $19681857^{\text {AL }}$, p. 2406-2408. In S. T. Williams, M. E. Sharpe, and J. B. Holt (ed.), Bergey's manual of systematic bacteriology, vol. 4. Williams and Wilkins, Baltimore.

33. Maggia, L., and J. Bousquet. 1994. Molecular phylogeny of the actinorhizal Hamamelidae and relationship with host promiscuity towards Frankia. Mol. Ecol. 3:459-467.

34. Mirza, M. S., W. M. Akkermans, and A. D. L. Akkermans. 1994. PCRamplified $16 \mathrm{~S}$ rRNA sequence analysis to confirm nodulation of Datisca cannabina L. by the endophyte of Coriaria nepalensis Wall. Plant Soil 160: 147-152.

35. Mirza, M. S., D. Hahn, and A. D. L. Akkermans. 1992. Isolation and characterization of Frankia strains from Coriaria nepalensis. Syst. Appl. Microbiol. 15:289-295.

36. Moran, N. A., M. A. Munson, P. Baumann, and H. Ishikawa. 1993. A molecular clock in endosymbiotic bacteria is calibrated using the insect hosts. Proc. R. Soc. Lond. B Biol. Sci. 253:167-171

37. Mullis, K. B., and F. A. Faloona. 1987. Specific synthesis of DNA in vitro via a polymerase-catalyzed chain reaction. Methods Enzymol. 155:335-350.

38. Murry, M. A., M. S. Fontaine, and J. G. Torrey. 1984. Growth kinctics and nitrogenase induction in Frankia sp. HFP Arl3 grown in batch culture. Plan Soil 78:61-78.

39. Nazaret, S., B. Cournoyer, P. Normand, and P. Simonet. 1991. Phylogenetic relationships among Frankia genomic species determined by use of amplified 16S rDNA sequences. J. Bacteriol. 173:4072-4078.

40. Nick, G., E. Paget, S. Simonet, A. Moiroud, and P. Normand. 1992. The nodular endophytes of Coriaria spp. form a distinct lineage within the genus Frankia. Mol. Ecol. 1:175-181.

41. Normand, P., B. Cournoyer, S. Nazaret, and P. Simonet. 1992. Analysis of a ribosomal rRNA operon in the actinomycete Frankia. Gene 111:119-124.

41a.Normand, P., and M. Lalonde. 1982. Evaluation of Frankia strains isolated from provenances of two Alnus species. Can. J. Microbiol. 28:1133-1142.

42. Ochman, H., and A. C. Wilson. 1987. Evolution in bacteria: evidence for a universal substitution rate in cellular genomes. J. Mol. Evol. 26:74-86.

43. Pommer, E. H. 1959. Uber die Isolierung des Endophyten aus den Wurzelknöllchen Alnus glutinosa Gaertn. und über erfolgreiche Re-Infektionsversuche. Ber. Dtsch. Bot. Ges. 72:138-150.

44. Rainey, F., and E. Stackebrandt. 1993. Phylogenetic evidence for the clas sification of Acidothermus cellulolyticus into the subphylum of actinomycetes. FEMS Microbiol. Lett. 108:27-30.

45. Rainey, F. A., P. Schumann, H. Prauser, R. Toalster, and E. Stackebrandt. 1993. Sporichthya polymorpha represents a novel line of descent within the order Actinomycetales. FEMS Microbiol. Lett. 109:263-268.

46. Saitou, R. R., and M. Nei. 1987. A neighbor-joining method: a new method for reconstructing phylogenetic trees. Mol. Biol. Evol. 44:406-425.

47. Simonet, P., M. Bosco, A. Moiroud, and P. Normand. 1994. Molecular characterization of Frankia microsymbionts from spore-positive and spore negative nodules in a natural alder stand. Appl. Environ. Microbiol. 60:13351341.

48. Simonet, P., A. Capellano, E. Navarro, R. Bardin, and A. Moiroud. 1984. An improved method for lysis of Frankia with achromopeptidase allows detec tion of new plasmids. Can. J. Microbiol. 30:1292-1295.

49. Simonet, P. P. Normand, A. Moiroud, and R. Bardin. 1990. Identification of Frankia strains in nodules by hybridization of polymerase chain reaction products with strain specific oligonucleotide probes. Arch. Microbiol. 153: 235-240.

50. Simonet, P., P. Normand, A. Moiroud, and M. Lalonde. 1985. Restriction enzyme digestion patterns of Frankia plasmids. Plant Soil 87:49-60.

51. Thomas, B. A., and R. A. Spicer. 1987. The evolution and paleobiology of land plants. Croom Helm, London.

52. Van Dijk, C. 1978. Spore formation and endophyte diversity in root nodules of Alnus glutinosa (L). Vill. New Phytol. 81:601-615.

53. Vobis, G. 1984. Actinoplanetes, p. 2418-2428. In N. R. Krieg and J. G. Holt (ed.), Bergey's manual of systematic bacteriology, vol. 1. Williams and Wilkins, Baltimore.

54. Woese, C. R., R. Gutell, R. Gupta, and H. F. Noller. 1983. Detailed analysis of the higher order structure of $16 \mathrm{~S}$-like ribosomal ribonucleic acids. Microbiol. Rev, 47:621-669. 